\title{
Risk Factors for Visual Field Progression in New- diagnosed Exfoliation Glaucoma Patients in Sweden.
}

Marcelo Ayala ( $\sim$ marcelo.ayala@vgregion.se)

University of Gothenburg

\section{Research Article}

Keywords: exfoliation glaucoma, visual field progression, risk factors, Sweden

Posted Date: January 5th, 2022

DOI: https://doi.org/10.21203/rs.3.rs-1217917/v1

License: (c) (i) This work is licensed under a Creative Commons Attribution 4.0 International License.

Read Full License 


\section{Abstract}

Purpose: The present study aimed to identify risk factors for visual field progression in new-diagnosed exfoliation glaucoma patients.

Methods: Prospective non-randomized cohort study. The study included patients with new-diagnosed exfoliation glaucoma. All patients were followed for at least three years with reliable visual fields. Both risk factors at inclusion and during the three years follow-up were considered. For inclusion, five reliable visual fields were needed. Exfoliation glaucoma was defined based on the European Glaucoma Society guidelines. Visual field evaluation was performed using the 24-2 strategy of the Humphrey Field Analysis. Outcomes: Visual field progression. Three different approaches were used: mean deviation (MD), visual field index (VFI), and guided progression analysis (GPA).

Results: The results were different in the three different models used (MD, VFI and GPA). The only variable that showed a significant association in the three models was age $(p=0.004 ; p=0.006 ; p=0.04)$.

Significant variables in two models were: IOP at diagnose $(p=0.02 ; p=0.04)$, IOP reduction in absolute terms $(p=0.006 ; p=0.003)$, IOP reduction in relative terms $(\%)(p=0.04 ; p=0.009)$ and number of medicines $(p=0.02 ; p=0.002)$. Significant variables in one model were: family history $(p=0.04)$, smoking $(p=0.03)$, cataract surgery $(p=0.04)$ and SLT treatment $(p=<0.001)$.

Conclusion: Exfoliation glaucoma is fast progressive glaucoma. Age at diagnosing must be considered. Significant IOP reduction must be achieved to slow down progress in exfoliation glaucoma. The use of SLT treatment should be advised in exfoliation glaucoma patients.

\section{Key Messages}

Risk factors for glaucoma development and progression have been described before. The present article showed that age was the most critical risk factor for progression in new-diagnosed exfoliation glaucoma patients in Sweden. Other risk factors were IOP at diagnosis, IOP reduction, and the number of medicines used.

\section{Background}

Glaucoma is an eye disease that affects the optic nerve. Usually, the visual fields are damaged, but in advanced cases can the disease even lead to blindness. Together with age-related macular degeneration, it is one of the most common causes of blindness in western countries [1]. A progressive loss of ganglion cells characterizes glaucoma. Unfortunately, no treatment has been discovered to cure the disease. The intrinsic causes of most glaucoma have not been found. However, several risk factors have been described. The most commonly cited risk factor for glaucoma development is increased intraocular pressure (IOP) [2]. There are several clinical manifestations of glaucoma; among them, the two most common clinical types in Sweden are primary open-angle (POAG) and exfoliation glaucoma (EXFG) [3]. 
Exfoliation glaucoma is secondary open-angle glaucoma characterized by a protein-based material that deposits in the eye's anterior chamber. The origin of this exfoliation material has not yet been discovered. This greyish material Exfoliation material has been isolated in different parts of the eye, but even other ocular and extraocular tissues showed the presence of this exfoliation material [4-7]. The deposits of exfoliation material at the trabecular meshwork occlude the pores of the trabecular meshwork diminishing the outflow of aqueous humor, thus increasing the IOP.

Risk factors for developing glaucoma have been well-defined for POAG but not so well for EXFG. According to previous studies, risk factors commonly associated with POAG were age, elevated IOP at baseline, lower central corneal thickness, and family history [8-11]. It's supposed that similar risk factors can apply for exfoliation glaucoma. However, genetic causes differed between POAG and EXFG [12]. Previous studies have also described risk factors for visual field deterioration in POAG. In general, age and IOP peaks were defined as risk factors for visual fields' damage $[8,10,11,13,14]$, and even exfoliation was cited as a risk factor for progression $[10,13]$. Interestingly, no evidence was found about risk factors for visual field deterioration in new-diagnosed exfoliation glaucoma patients.

As described above, glaucoma is a progressive disease. Glaucoma progression is estimated by visual field deterioration. How progression develops varies significantly among individuals $[15,16]$. Therefore, it's essential to determine the rate of progression in every patient to choose the best treatment modality available. The European Glaucoma Guidelines [17] recommends 5-6 visual fields in 2 years after diagnosing glaucoma to estimate progression. The Swedish Glaucoma Guidelines [18] recommend 5-6 visual fields in 3 years. This strategy was judged to be more realistic to how the Swedish Health System works. Swedish patients' "golden standard" for glaucoma progression analysis is still visual field testing using computer-assisted perimetry.

The purpose of the present study was to identify risk factors for visual field progression in new-diagnosed exfoliation glaucoma patients. Both risk factors at diagnosing and risk factors during the three-year control period were studied.

\section{Methods}

The present study was a prospective non-randomized cohort study. All patients with new-diagnosed exfoliation glaucoma were recruited. The inclusion period was from 1st January 2012 till 31 st December 2016 (five years). All patients attended the Ophthalmology Department at Skaraborg's Hospital. The Ophthalmology Department at the Skaraborg's Hospital takes care of around 250000 inhabitants.

\section{Inclusion criteria:}

Patients were suffering from new-diagnosed exfoliation glaucoma. The definition of exfoliation glaucoma was an untreated IOP of $\geq 21 \mathrm{~mm} \mathrm{Hg}$, open anterior chamber angle, glaucomatous visual field defect (at least two repeatable Humphrey 24-2), and glaucomatous optic nerve damage; all together with the presence of exfoliation material, according to the definition of the European Glaucoma Society [17]. 


\section{Exclusion criteria:}

1. Patients who could not perform reliable visual fields at the beginning of the study and/or could perform at least five reliable visual fields three years after the exfoliation glaucoma diagnosis. The following criteria were used to define reliable visual fields: false positives $\leq 15 \%$ and/or false negatives $\leq 20 \%$ and/or fixation losses $\leq 30 \%$.

2. Patients with advanced glaucoma defined as mean deviation $(M D) \geq 18 \mathrm{~dB}$ and/or visual field index $(\mathrm{VFI}) \leq 40 \%$ were excluded. These patients were excluded to avoid "floor effects" in which further loss of visual field defects can no longer be detected $[19,20]$.

3. Patients needing glaucoma surgery during the follow-up period. Uncomplicated cataract surgery and/or selective laser treatment (SLT) were not considered exclusion criteria.

4. Patients suffering from another eye disease could modify visual fields under the study period like central vein occlusion, retinal detachment, etc.

5. Patients were dropping out of the three years control period due to different reasons like dementia, moving to another city, etc.

Risk factors studied at diagnosis were age, sex, unilateral/bilateral glaucoma, visual acuity, refractive errors, IOP, central corneal thickness (CCT), gonioscopy: anterior chamber depth and pigmentation, cupdisc ratio (C/D), diabetes, smoking, hypertension, migraine and family history for glaucoma.

Risk factors studied under the three years were: IOP reduction in absolute values, IOP reduction in relative values, SLT treatment, cataract operation, and the number of medications.

Every patient was detailed examined at inclusion. All patients were referred to the Ophthalmology Department due to high IOP ( $\geq 21 \mathrm{mmHg}$ ) detected by an optician/optometrist. Age was registered as age at glaucoma diagnosis. Sex was registered as male/female. Unilateral/bilateral presence of glaucoma was recorded. In the case both eyes were suffering glaucoma, one eye was chosen at random.

Visual acuity was measured using a Snellen chart. Refractive errors were registered based on the consultation chart sent by the optician/optometrist. For more straightforward calculations, the aspheric equivalent was calculated. The IOP was measured using a Goldman's applanation tonometer. The central corneal thickness (CCT) was measured using an ultrasound device (Tomey Pachymetry; Tomey Corp, Nagoya 451-0051, Japan). The average value of seven measurements was automatically calculated. To assess trabecular meshwork, gonioscopy was performed using a goniolens with undilated pupils. According to Shaffer's system, the anterior chamber was studied and classified as deep (0-4). The trabecular meshwork was studied regarding pigmentation and ranked 0-3. The patient's pupils were then dilated with 2.5\% Phenylephrine and Tropicamide 0.5\% (Bausch \& Lomb UK Ltd, 106 London RoadKingston-upon-Thames-Surrey-KT2 6TN-England). Eyes were classified as exfoliation if there was evidence of exfoliation material on the pupil, lens, or the angle with dilated pupils. The status of the optic nerve was assessed using a 90-D lens, and the average vertical cupping was recorded as the cup-to-disc ratio $(C / D)$. 
Furthermore, all patients answer a questionnaire with questions regarding hypertension, smoking, diabetes, migraine, and family history of glaucoma. Hypertension was defined as "using medicines against high blood pressure" (Yes/No). Smoking was described as "smoked more than 50 cigarettes in your life" (Yes/No). Diabetes was defined as "using medicines against diabetes" (Yes/No). Migraine was defined as "suffered from migraine" (Yes/No). Family history for glaucoma was defined as having near relative suffering from glaucoma. Near relative was considered: father and/or mother and/or siblings. The answer was registered as "Yes/No."

Risk factors for progression under the three years were measured as follows: IOP reduction in absolute values was measured as the IOP three years after diagnosis (IOP at diagnosis-IOP at three years). The relative IOP reduction was calculated as a percentage (\%).

The SLT treatments were measured as present or absent per each patient. An uneventful cataract operation was not a reason for exclusion and was counted as present or absent. Eye-drops at the end of the three years were measured as the number of medicines (compounds) and not as the number of bottles.

The endpoint of the study was the visual field's progression. All patients were examined using Humphrey Field Analysis (Carl Zeiss, Carl-Zeiss-Straße 22, 73447 Oberkochen, Germany) using the software threshold 24-2. Three different methods for the assessment of visual field progression were used.

The first method was based on the "mean deviation" (MD) visual field parameter. The difference in MD values from the beginning to the end of the study was calculated. Higher values indicated higher progression. The MD values were chosen since several studies still use MD as an indicator of progression $[16,21,22]$. Cataract development can also modify MD values.

The second method used was the glaucoma progression analysis based on the "visual field index" (VFI). The device calculated the VFI and performed a regression analysis calculating the "rate of progression" (ROP). The ROP was calculated automatically by the device as the amount of VFI deterioration (\%)/year. The ROP calculation is also called a "trend analysis."

The third progression analysis used was the guided progression analysis (GPA). The GPA is also included in the device and performed automatically (GPA Alert). The GPA is different from the previous one (ROP). The GPA Alert analysis is called an "event analysis." The machine compares every single point to prior examinations. The GPA alert results can be no, possible or likely progression. For analysis purposes in this study, the results were evaluated as "no progression" or "progression," which included both "possible" and "likely" progression.

\section{Statistics}

SPSS (IBM, 1 New Orchard Road Armonk, NY 10504, USA) software was used for statistical analysis. The variables were tested in a "2 step manner". The first step, to test the association between variables and progression, a univariate linear regression analysis was done for continuous endpoints variables (MD and 
VFI). Then, for the dichotomous endpoint (GPA), a univariate logistic regression analysis was performed. The variables that showed a significant association in the univariate analysis were retested using multivariate analysis. Again, a multivariate linear regression analysis was used when the endpoints were continuous (MD and VFI); meanwhile, a multivariate logistic regression analysis was performed when the endpoint was dichotomous (GPA). The significance level was set at 0.05 .

\section{Results}

Totally, 91 patients were included in this study. Fourteen $(n=14)$ patients were excluded. The reasons for exclusion were $n=4$ due to very damaged visual fields initially, $n=5$ did not attend the check-up visits and/or could not perform reliable visual fields, $n=3$ developed other eye diseases during the three years, and $n=2$ was glaucoma operated. The average age at inclusion was 71.41 ( \pm 7.52$)$ years (range: 47-88). Regarding sex distribution, $n=49(53.85 \%)$ was female and $n=42(46.15 \%)$ was male (Chi-square; $p=0.46)$. Glaucoma was presented unilateral in $n=62(68.13 \%)$ and bilateral in $n=29(31.87 \%)$ (Chi-square; $p=$ $<0.001)$. At diagnosis, the average IOP value was $33.14 \mathrm{mmHg}( \pm 6.66)$ (range: $22-55)$. Visual acuity at diagnosis was $0.82( \pm 0.25)$. Refractive errors showed to be $0.47 \mathrm{D}( \pm 1.63)$, range: $-4.25 /+3.25 \mathrm{D}$. The average CCT values were $541.6 \mu \mathrm{m}( \pm 32.97)$. Gonioscopy at diagnosis showed an average anterior chamber depth of $3.13( \pm 0.61)$, pigmentation of the trabecular meshwork was $2.47( \pm 0.64)$. The $C D$ ratio at diagnosis was $0.73( \pm 0.17)$.

Patients that reported hypertension were $n=50(55 \%)$, meanwhile $n=41(45 \%)$ reported no hypertension (Chi-square; $p=0.34)$. Smoking was reported by $n=41(45 \%)$ and no-smoking was reported by $n=50(55 \%)$ of patients (Chi-square; $p=0.34)$. Diabetes was reported by $n=14(15.38 \%)$ meanwhile no diabetes was reported by $n=77(84.62 \%)$ (Chi-square; $p=<0.001)$. The presence of migraine was reported in only $n=6$ $(6.6 \%)$ of cases, meanwhile $n=85(93.4 \%)$ reported no migraine (Chi-square; $p=<0.001)$. Family history for glaucoma was reported by $n=39(42.85 \%)$ meanwhile $n=52(57.15 \%)$ reported no family history (Chisquare, $p=0.17$ ).

After three years, the average IOP was $17.88 \mathrm{mmHg}( \pm 2.99)$. The absolute IOP reduction at three years was $15.35 \mathrm{mmHg}( \pm 7.38)$. The relative IOP reduction at three years was an average of $47.28 \%( \pm 14.28)$. The number of cataract patients operated on during the three-years follow-up period was $n=14$. The number of patients treated with SLT was $n=16$. The number of medicines at three years follow-up was $2.66( \pm 0.89)$.

Regarding visual fields parameters at baseline, the MD was $-6.41 \mathrm{~dB}( \pm 5.01)$, and the VFI: was $85.4 \%$ ( \pm 14.64$)$. After three years' follow-up, the visual field parameters were: MD $-9.95 \mathrm{~dB}( \pm 6.31)$, and the VFI was $75.61 \%( \pm 19.12)$. The rate of progression based on VFI was $-2.60 \%( \pm 2.38) /$ year. The G.P.A. showed that $n=55$ progressed and $n=36$ not progressed (Chi-square; $p=0.04$ ).

The risk factors at diagnosis that showed a significant association with visual field deterioration differed in the three models studied. In the MD model, the variables that showed a significant association were 
age, IOP reduction in absolute numbers, and cataract surgery during the follow-up period. Please see table 1.

In the VFI model, the variables associated with progress were age, family history, smoking, IOP at diagnosis, IOP reduction (both the absolute and the relative values), the number of medicines, and the SLT treatment. Please see table 2.

In the GPA model, the variables associated with progress were age, IOP at diagnosis, IOP reduction in relative values, and the number of medicines. Please see table 3.

The only variable that showed significant association in the three different models was age. The IOP at diagnosis, IOP reduction in absolute values, and the IOP reduction in relative values showed a significant association in two of the three models studied. Family history for glaucoma, smoking, and SLT treatment showed a significant association only in the VFI model. A summary of the significantly associated variables has been included for better visualization. Please see table 4 .

\section{Discussion}

The present study tried to elucidate the risk factors for visual field deterioration in a cohort of newdiagnosed exfoliation glaucoma patients in Sweden. The risk factors studied were recorded both at diagnosis and during the three-year follow-up period.

Previous studies have extensively described risk factors for developing glaucoma [8-11]. In addition, risk factors for progression in glaucoma damage have also been described before [23]. Interestingly, exfoliation seems to be a risk factor for fast progression in previous studies compared with other glaucoma types. However, evidence about if the described risk factors apply for new-diagnosed exfoliation glaucoma patients is lacking. In several previously published studies, exfoliation glaucoma patients were excluded due to the low prevalence of exfoliation glaucoma in other populations and because the disease develops differently. Exfoliation glaucoma patients showed a more aggressive and fast development of visual field damage than primary-open glaucoma patients [24]. At our Department, around $60 \%$ of all new-diagnosed glaucoma patients suffer from exfoliation glaucoma, making results from this study very interesting from the clinical point of view.

Age showed to be a common risk factor for progression in the three models studied. The average age at inclusion was $71.41( \pm 7.52)$ years. This result accords with previous studies in which the average age at glaucoma diagnosis was around 70 years old [25]. Only three patients were excluded from the study due to their disability to perform visual fields. All of them were about 70 years old, meaning that no patient was indirectly excluded due to age. Considering the $\beta$ coefficients at the multivariate linear regression analysis, the MD values increased by $0.092 \mathrm{~dB} /$ year. The MD increase can be due to age (cataract) or glaucoma progression. However, age was also positively associated with visual field progress when considering the rate of progression (ROP). ROP is based on the visual field index (VFI) which corrects for cataracts and evaluates more accurate glaucoma damage. The $\beta$ coefficient at the multivariate linear 
regression analysis showed an ROP deterioration of $0.053 \% /$ year. This increased ROP value means that a patient diagnosed with glaucoma at 80 years old had a faster visual field deterioration than a patient diagnosed at 70 years old. The average ROP was $2.60 \% /$ year for the whole cohort. In the hypothetical case that a patient aged 70 progressed at $2.60 \%$ /year, 80 would progress at $2.65 \% /$ year. These results must be interpreted with caution. The results apply to the range studied, let's say 47-88 years.

Furthermore, the model was linear; this assumption can be suitable for the years studied but probably not for older ages. Other models, like exponential, probably suit better with reality in higher ages. Further studies are needed to clarify the best models for different age groups. The study showed increased progress with increased age. The increased progress with age could be that the number of ganglion cells diminished with age, so the visual fields deteriorate faster as age increases.

Several previous evidence described the IOP as the most critical risk factor for glaucoma development. The present study showed an association between IOP at diagnosis, IOP reduction in absolute and relative values, and visual fields' deterioration in new-diagnosed exfoliation glaucoma patients. The average IOP was high at diagnosing, $33.14 \mathrm{mmHg}( \pm 6.66)$. Exfoliation glaucoma is a high IOP glaucoma. Exfoliation material obstructs the trabecular meshwork producing high IOP levels. The average ROP in the cohort was $2.60 \%$ /year during the three years' follow-up. Based on the results from the multivariate analysis for ROP ( $\beta$ coefficient), every $\mathrm{mmHg}$ IOP in the baseline represented an increased ROP of 0.05 . Theoretically, this means that a patient with a baseline IOP of $25 \mathrm{mmHg}$ and an ROP of $2.60 \%$ /year would progress at $2.65 \%$ year if the IOP were $35 \mathrm{mmHg}$ or at $2.70 \% /$ year if the IOP was of $45 \mathrm{mmHg}$. As pointed out above, this extrapolation must be taken with caution. It probably applies for the IOP levels registered in this study: $22-55 \mathrm{mmHg}$ and probably not for other IOP levels.

Furthermore, the model assumed that the relationship between IOP values and ROP is linear. This is not known; probably each mmHg has a different impact on ROP if the IOP is around $30 \mathrm{mmHg}$ than if it is around $50 \mathrm{mmHg}$. Further studies are needed to clarify this issue.

The IOP reduction is another interesting issue to discuss. The present study included both an absolute and a relative value for IOP reduction after diagnosis. Patients included in this study were followed and treated according to our guidelines for treatment and follow-up of glaucoma patients. All patients got what is called "target IOP" after diagnosis. This "target IOP" is an uncertain IOP value that usually is around $18-20 \mathrm{mmHg}$, and it's established by the ophthalmologist who made the diagnosis. According to our guidelines, three years after follow-up with at least 5-6 reliable visual fields, this "target IOP" must be re-evaluated. In the present study, the IOP three years after follow-up was $17.88 \mathrm{mmHg}( \pm 2.99)$. Even if this value can be considered acceptable, $2 / 3$ of patients showed progression in the GPA analysis. Therefore, it seems that the "target IOP" should be lower than $18 \mathrm{mmHg}$, probably around $16 \mathrm{mmHg}$ (or even lower) to diminish the visual field's deterioration. A similar approach can be made related to the relative IOP reduction. A common assumption is that a $20 \%$ IOP reduction is enough to stop the progression of the disease [26]. In the present study, the average IOP reduction was $47.28 \%( \pm 14.28)$. However, 2/3 of glaucoma patients showed progression according to the GPA analysis. The aggressive 
nature of these new-diagnosed exfoliation glaucoma patients probably needs a higher relative IOP reduction to stop the progress of the disease.

The absolute IOP reduction showed to be significantly associated in the MD and the VFI but not in the GPA model. Meanwhile, the relative IOP reduction was significantly associated with the VFI and the GPA but not the MD model. This means that both ways to control IOP reduction are needed. However, in our clinical practice, we usually forget the importance of taking care of both the absolute and the relative IOP reduction. We usually focus more on the absolute values, and sometimes we feel that it is difficult to find the IOP value at the diagnosis and calculate the relative IOP reduction. The present study showed the importance of checking both values to establish a more accurate level of IOP that can stop the visual field's deterioration.

Three variables showed a significant correlation with visual field progression only in the VFI model. The mentioned variables were family history, smoking, and SLT. Family history for glaucoma was measured in the present study as present or absent. Only closest relatives were considered; mother and/or father and/or siblings. The information was self-reported, reporting bias might be admitted. Family history for glaucoma was reported by $n=39(42.85 \%)$ meanwhile $n=52(57.15 \%)$ reported no family history (Chisquare, $p=0.17$ ). Most of the patients were born in Sweden, and their parents were also born in Sweden. Only two patients had Finnish parents. Genetic mechanisms behind exfoliation glaucoma have been described in previous studies [27]. However, no previous evidence regarding family history and a poorer prognostic for glaucoma has been found.

Smoking has been described among risk factors for glaucoma development [28]. In the present study, smoking was measured as present or absent based on a question: "Did you smoke more than 50 cigarettes in your life?" Also, this question was self-reported, addressing the possibility of reporting bias. Interestingly, a quite high number of patients reported that they have smoked $(n=41 ; 45 \%)$. Smoking is not so common among the new generations in Sweden. Furthermore, the study did not ask if the patients performed "snuff" (chewing moist powder tobacco), common in Sweden. The increased visual field deterioration among smokers can be explained by decreased blood circulation in small vessels due to nicotinamide effects.

The SLT was also recorded as present or absent to facilitate the analysis. Interestingly, the association was negative; let's say that patients treated with SLT showed a lower progression according to the VFI model. The decision to treat or not to treat with SLT was based on the clinicians that met the patients. SLT treatment has shown promising results in exfoliation glaucoma patients [29]. However, the number of patients treated with SLT in the present study was relatively low $(n=16)$. Based on the current study results, it's possible to recommend SLT often to exfoliation glaucoma patients.

The number of medicines was also associated with visual field progression in the present study. In the VFI model, the association was negative; let's say an increased number of medicines rendered a lower progression. At three years of follow-up, the patients were treated on $2.66( \pm 0.89)$ drugs. Based on the $\beta$ coefficient of the multivariate linear regression, each medicine reduced the ROP by $0.32 \% / y e a r$. The 
higher number of drugs induced a higher reduction in the IOP, thus diminishing progression. It's also possible that medications can reduce progression by other mechanisms apart from IOP reduction (neuroprotection).

The GPA (dichotomous) model also shows the relationship between the number of medicines and progression was also demonstrated in the GPA (dichotomous) model. The logistic regression analysis showed a significant association $(p=0.002)$ between the number of medications and the detection of progress/no progress of the visual fields in the GPA. A posthoc analysis showed that the average amount of medicines in the no progression group was $n=2.1 \pm 0.8$; meanwhile, in the progression group was $n=3$ \pm 0.7 . The estimated Odds Ratio (OR) coming from the regression analysis was 4.79 . This means an increased risk of being treated with more medicines if you are suffering from progressing glaucoma than if your glaucoma is not progressing. A posthoc analysis was performed to clarify the issue. The patients were divided into groups 1: 1 or 2 medicines and group 2: 3 or 4 (only one patient was treated with five medications and was excluded). Then an OR was measured between the two groups showing a value of 8.47. Therefore, individuals with progressive glaucoma are eight times more likely to be treated with 3 or 4 medicines than individuals that suffer from non-progressive glaucoma. This is quite logical during the follow-up period if the ophthalmologist suspected progression wanted to decrease IOP even more. Also, it must be considered that high IOP at the diagnosis will increase progression itself and require an increased number of medications.

Cataract surgery was performed in $n=14$ patients during the three years follow-up period. At the baseline, $\mathrm{n}=23$ patients were already cataract operated. Interestingly, cataract surgery showed association with progression only in the MD-based model. Cataract surgery improved the values in MD in the visual fields. The improvement of visual fields due to cataract surgery was not observed in the two other models (VFI and GPA). Nowadays, it's a general agreement not to use MD to evaluate glaucoma progression since cataract surgery can alter the parameter. MD was included in the present study because it has been included in several studies before. [25] The other reason is that the most common glaucoma classification (Hodapp's classification) is MD values [30]. The included patients in this study mainly belonged to the early and moderate glaucoma groups.

The study has certain limitations. As pointed out above, most patients belonged to early ( $M D=0-5.9 \mathrm{~dB})$ and moderate ( $M D=6-12 \mathrm{~dB}$ ) glaucoma groups. Very few patients with advanced glaucoma were included. Patients were excluded if they showed an $M D \geq 18 \mathrm{~dB}$ and/or VFI $\leq 40 \%$. This criterium was chosen to avoid "floor effects" in which further loss of visual field defects can no longer be detected [19, 20]. This can be a limitation; unfortunately, advanced glaucoma patients cannot be followed with visual fields. Progression assessment would not be accurate when the visual fields are so damaged.

Another limitation of the study is that no morphological assessment of the optic nerve was done. The only parameter included was the cup/disc ratio (C/D), but this is a quite subjective manner of optic nerve assessment. In addition, the use of optic nerve OCT was not so common when the study began. Nowadays, OCT technology is part of the tools for glaucoma follow-up at our Department. However, it 
seems that OCT also has "floor effects" for advanced glaucoma patients [31]. Still, there is no consensus about if morphology or function is the best to evaluate glaucoma progression [32].

In conclusion, the present study showed that age was the most critical risk factor for progression in newdiagnosed exfoliation glaucoma patients in Sweden. The other factors to be considered are IOP at diagnosis and IOP reduction both in absolute and relative number. Factors with lower impact in progression were positive family history, smoking, and SLT. Therefore, SLT treatment should be recommended in this patient group to slow down progression. Exfoliation glaucoma is an aggressive form of glaucoma that must be monitored often to avoid advanced visual field damage and blindness.

\section{Declarations}

Competing interests: No funding was received for conducting the present study. The author has no financial or proprietary interests in any material discussed in this article.

Author's contribution: There is just one author of this article.

Compliance with Ethical Standards: This study was performed in line with the principles of the Declaration of Helsinki. Approval was granted by the Ethics Committee of Gothenburg's University (DN: 119-12.).

Consent to participate: Informed consent was obtained from all individual participants included in the study.

\section{References}

1. Resnikoff, S., et al., Global data on visual impairment in the year 2002. Bull WHO, 2004. 82(11): p. 844-851.

2. Leske, M.C., et al., Factors for glaucoma progression and the effect of treatment: the early manifest glaucoma trial. Arch Ophthalmol, 2003. 121(1): p. 48-56.

3. Aström, S., H. Stenlund, and C. Lindén, Incidence and prevalence of pseudoexfoliations and openangle glaucoma in northern Sweden: II. Results after 21 years of follow-up. Acta Ophthalmologica Scandinavica, 2007. 85(8): p. 832-837.

4. Ritch, R. and U. Schlotzer-Schrehardt, Exfoliation syndrome. Surv Ophthalmol, 2001. 45(4): p. 265315.

5. Dewundara, S. and L.R. Pasquale, Exfoliation syndrome: a disease with an environmental component. Curr Opin Ophthalmol, 2015. 26(2): p. 78-81.

6. Ritch, R., Ocular and systemic manifestations of exfoliation syndrome. J Glaucoma, 2014. 23(8 Suppl 1): p. S1-8.

7. Pasquale, L.R., et al., Exfoliation syndrome: assembling the puzzle pieces. Acta Ophthalmol, 2016. 94(6): p. e505-12. 
8. Zhou, K., et al., [Risk factors for visual field loss progression in patients with primary open-angle glaucoma in Wenzhou area]. Zhonghua Yan Ke Za Zhi, 2019. 55(10): p. 777-784.

9. Mowatt, L., Risk Factors for Rapid Glaucoma Disease Progression. Am J Ophthalmol, 2018. 186: p. 170-171.

10. Chan, T.C.W., et al., Risk Factors for Rapid Glaucoma Disease Progression. Am J Ophthalmol, 2017. 180: p. 151-157.

11. Actis, AG, et al., Risk Factors for Primary Open Angle Glaucoma (POAG) Progression: A Study Ruled in Torino. Open Ophthalmol J, 2016. 10: p. 129-39.

12. Ghaffari Sharaf, M., K.F. Damji, and L.D. Unsworth, Recent advances in risk factors associated with ocular exfoliation syndrome. Acta Ophthalmol, 2020. 98(2): p. 113-120.

13. Kim, J.H., et al., Risk Factors for Fast Visual Field Progression in Glaucoma. Am J Ophthalmol, 2019. 207: p. 268-278.

14. Hung, K.H., C.Y. Cheng, and C.J. Liu, Risk factors for predicting visual field progression in Chinese patients with primary open-angle glaucoma: A retrospective study. J Chin Med Assoc, 2015. 78(7): p. 418-23.

15. Baek, S.U., et al., Long-Term Follow-up on Glaucoma Patients With Initial Single-Hemifield Defect: Progression Patterns and Associated Factors. J Glaucoma, 2019. 28(12): p. 1041-1047.

16. Salonikiou, A., et al., Tolerable rates of visual field progression in a population-based sample of patients with glaucoma. Br J Ophthalmol, 2018. 102(7): p. 916-921.

17. European Glaucoma Society Terminology and Guidelines for Glaucoma, 4th Edition - Chapter 2: Classification and terminology Supported by the EGS Foundation: Part 1: Foreword; Introduction; Glossary; Chapter 2 Classification and Terminology. Br J Ophthalmol, 2017. 101(5): p. 73-127.

18. Heijl, A., et al., The Glaucoma Guidelines of the Swedish Ophthalmological Society. Acta Ophthalmol Suppl (Oxf), 2012(251): p. 1-40.

19. Wall, M., et al., Repeatability of automated perimetry: a comparison between standard automated perimetry with stimulus size III and V, matrix, and motion perimetry. Invest Ophthalmol Vis Sci, 2009. 50(2): p. 974-9.

20. Nguyen, A.T., et al., Detecting Glaucoma Progression Using Guided Progression Analysis with OCT and Visual Field Assessment in Eyes Classified by International Classification of Disease Severity Codes. Ophthalmology Glaucoma, 2019. 2(1): p. 36-46.

21. Liebmann, K., CG De Moraes, and J.M. Liebmann, Measuring Rates of Visual Field Progression in Linear Versus Nonlinear Scales: Implications for Understanding the Relationship Between Baseline Damage and Target Rates of Glaucoma Progression. J Glaucoma, 2017. 26(8): p. 721-725.

22. Berchuck, S.I., S. Mukherjee, and F.A. Medeiros, Estimating Rates of Progression and Predicting Future Visual Fields in Glaucoma Using a Deep Variational Autoencoder. Sci Rep, 2019. 9(1): p. 18113. 
23. Zhang, X., et al., Predictive Factors for the Rate of Visual Field Progression in the Advanced Imaging for Glaucoma Study. Am J Ophthalmol, 2019. 202: p. 62-71.

24. Ayala, M., Comparison of visual field progression in new-diagnosed primary open-angle and exfoliation glaucoma patients in Sweden. BMC Ophthalmol, 2020. 20(1): p. 322.

25. Heijl, A., et al., Natural history of open-angle glaucoma. Ophthalmology, 2009. 116(12): p. 2271-2276.

26. World Glaucoma Association, C., Guidelines on Design and Reporting of Glaucoma Surgical Trials, in Guidelines on Design and Reporting of Glaucoma Surgical Trials (WGA). T.M. Shaarwy, M.B. Sherwood, and F. Grehn, Editors. 2009, Kugler Publication.

27. Thorleifsson, G., et al., Common sequence variants in the LOXL1 gene confer susceptibility to exfoliation glaucoma. Science, 2007. 317(5843): p. 1397-1400.

28. Pérez-de-Arcelus, M., et al., Smoking and incidence of glaucoma: The SUN Cohort. Medicine (Baltimore), 2017. 96(1): p. e5761.

29. Ayala, M. and E. Chen, Comparison of selective laser trabeculoplasty (SLT) in primary open angle glaucoma and pseudoexfoliation glaucoma. Clin Ophthalmol, 2011. 5: p. 1469-73.

30. Hodapp, E., R.K. Parrish, and D.R. Anderson, Clinical decisions in glaucoma. 1993, St. Louis, Mo. ; London: Mosby.

31. Abu, S.L., I. Marin-Franch, and L. Racette, Detecting Progression in Patients With Different Clinical Presentations of Primary Open-angle Glaucoma. J Glaucoma, 2021. 30(9): p. 769-775.

32. Vianna, JR and BC. Chauhan, How to detect progression in glaucoma. Prog Brain Res, 2015. 221: p. $135-58$.

\section{Tables}

Table 1 Significant variables tested in the univariate and the multivariate linear regression analysis using the MD difference as endpoint. 


\begin{tabular}{|llll|}
\hline Variables & Univariate & \multicolumn{2}{l|}{ Multivariate } \\
\hline & P-values & P-values & $\beta$ coef. \\
\hline Age & $0.034^{*}$ & $0.004^{*}$ & 0.092 \\
\hline Smoking & $0.005^{\star}$ & 0.35 & NA \\
\hline Gonioscopy (Shaffer) & $0.03^{*}$ & 0.14 & NA \\
\hline IOP diagnose & $0.001^{*}$ & 0.30 & NA \\
\hline Cataract surgery & $<0.001^{*}$ & $0.04^{*}$ & -1.24 \\
\hline IOP reduction absolute & $<0.001^{*}$ & $0.003^{*}$ & -0.013 \\
\hline IOP reduction relative & $<0.001^{*}$ & 0.08 & NA \\
\hline Amount of medicines & $0.023^{*}$ & 0.55 & NA \\
\hline SLT & $<0.001^{*}$ & 0.09 & NA \\
\hline
\end{tabular}

(*) Significant values. MD: Mean deviation. IOP: Intraocular pressure. SLT: Selective Laser Treatment. NA: Non-applicable.

Table 2 Significant variables tested in the univariate and the multivariate linear regression analysis using the VFI (ROP) as endpoint.

\begin{tabular}{|llll|}
\hline Variables & Univariate & \multicolumn{2}{l|}{ Multivariate } \\
\hline Age & P-values & P-values & $\beta$ coef. \\
\hline Family History & $0.004^{\star}$ & $0.006^{*}$ & 0.053 \\
\hline Smoking & $0.02^{\star}$ & $0.04^{\star}$ & 0.129 \\
\hline VA & $0.005^{\star}$ & $0.03^{\star}$ & 0.145 \\
\hline Gonioscopy (Shaeffer) & $0.026^{\star}$ & 0.76 & NA \\
\hline IOP at diagnose & $0.034^{\star}$ & 0.54 & NA \\
\hline IOP reduction absolute values & $<0.001^{\star}$ & $0.006^{*}$ & -0.070 \\
\hline IOP reduction relative values & $0.025^{\star}$ & $0.04^{*}$ & -0.022 \\
\hline Number of medicines & $<0.001^{*}$ & $0.02^{*}$ & -0.32 \\
\hline SLT & $<0.001^{*}$ & $<0.001^{*}$ & -1.28 \\
\hline
\end{tabular}


(*) Significant values. VFI: Visual field index. ROP: Rate of progression. VA: Visual acuity. IOP: Intraocular pressure. SLT: Selective Laser Treatment. NA: Non-applicable.

Table 3 Significant variables tested in the univariate and the multivariate logistic regression analysis using the GPA (dichotomous: progress/no progress) as endpoint.

\begin{tabular}{|llll|}
\hline Variable & Univariate & \multicolumn{2}{l|}{ Multivariate } \\
\hline & P-values & P-values & Exp B \\
\hline Age & $0.02^{\star}$ & $0.04^{\star}$ & 1.12 \\
\hline Family History & $0.03^{\star}$ & 0.26 & $\mathrm{NA}$ \\
\hline Smoking & $0.04^{\star}$ & 0.76 & $\mathrm{NA}$ \\
\hline IOP diagnose & $0.02^{\star}$ & $0.04^{\star}$ & 1.23 \\
\hline IOP reduction absolute & $<0.0001^{*}$ & 0.14 & $\mathrm{NA}$ \\
\hline IOP reduction relative & $<0.0001^{\star}$ & $0.009^{\star}$ & 1.07 \\
\hline Amount of medicines & $<0.0001^{*}$ & $0.002^{\star}$ & 4.79 \\
\hline
\end{tabular}

(*) Significant values. GPA: Guided progression analysis. VA: visual acuity. IOP: Intraocular pressure. NA: Non-applicable.

Table 4 Summary of the significant variables tested in the multivariate analysis according to the endpoints. 


\begin{tabular}{|llll|}
\hline & MD & VFI & GPA \\
\hline Age & $\mathrm{X}$ & $\mathrm{X}$ & $\mathrm{X}$ \\
\hline Family History & & $\mathrm{X}$ & \\
\hline Smoking & & $\mathrm{X}$ & \\
\hline Cataract surgery & $\mathrm{X}$ & & \\
\hline IOP at diagnose & & $\mathrm{X}$ & $\mathrm{X}$ \\
\hline IOP reduction absolute & $\mathrm{X}$ & $\mathrm{X}$ & \\
\hline IOP reduction relative & & $\mathrm{X}$ & $\mathrm{X}$ \\
\hline Amount of medicines & & $\mathrm{X}$ & $\mathrm{X}$ \\
\hline SLT & & $\mathrm{X}$ & \\
\hline
\end{tabular}

MD: Mean deviation. VFI: Visual field index. GPA: Guided progression analysis. IOP: Intraocular pressure. 